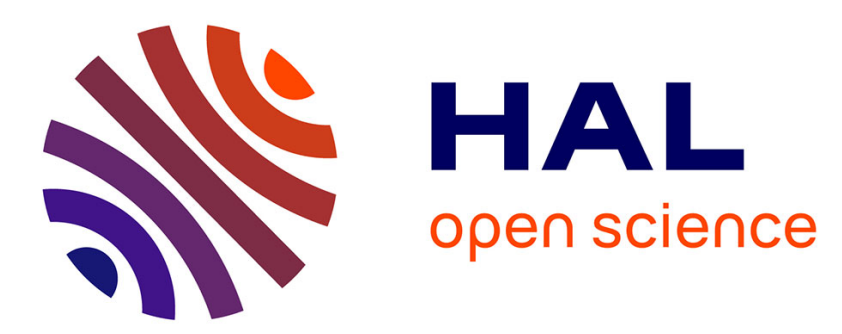

\title{
Evaluation of morphological representative sample sizes for nanolayered polymer blends
}

\author{
Adrien Bironeau, Justin Dirrenberger, Cyrille Sollogoub, Guillaume \\ Miquelard-Garnier, Sébastien Roland
}

\section{To cite this version:}

Adrien Bironeau, Justin Dirrenberger, Cyrille Sollogoub, Guillaume Miquelard-Garnier, Sébastien Roland. Evaluation of morphological representative sample sizes for nanolayered polymer blends. Journal of Microscopy, 2016, 264 (1), pp.48-58. 10.1111/jmi.12415 . hal-01383497

\section{HAL Id: hal-01383497 \\ https://hal.science/hal-01383497}

Submitted on 18 Oct 2016

HAL is a multi-disciplinary open access archive for the deposit and dissemination of scientific research documents, whether they are published or not. The documents may come from teaching and research institutions in France or abroad, or from public or private research centers.
L'archive ouverte pluridisciplinaire HAL, est destinée au dépôt et à la diffusion de documents scientifiques de niveau recherche, publiés ou non, émanant des établissements d'enseignement et de recherche français ou étrangers, des laboratoires publics ou privés. 


\title{
Evaluation of morphological representative sample sizes for nanolayered polymer blends
}

\author{
A. BIRONEAU, J. DIRRENBERGER, C. SOLlOGOUB, G. MIQUELARD-GARNIER \& S. ROLAND \\ PIMM, Arts et Métiers-ParisTech/CNAM/CNRS UMR 8006, 151 bd de l'Hôpital, Paris, France
}

Key words. Atomic force microscopy, coextrusion, multilayer structure, nanolaminate, nanolayered film, nanostructured material, polymer blend, representative volume element.

\section{Summary}

The size of representative microstructural samples obtained from atomic force microscopy is addressed in this paper. The case of an archetypal one-dimensional nanolayered polymer blend is considered. Image analysis is performed on micrographs obtained through atomic force microscopy, yielding statistical data concerning morphological properties of the material. The variability in terms of microstructural morphology is due to the thermomechanical processing route. The statistical data is used in order to estimate sample size representativity, based on an asymptotic relationship relating the inherent point variance of the indicator function of one material phase to the statistical, size-dependent, ensemble variance of the same function. From the study of nanolayered material systems, the statistical approach was found to be an effective mean for discriminating and characterizing multiple scales of heterogeneity.

\section{Introduction}

The question of representativity has been a topic of interest in scientific communities for half a century, especially in the field of materials science, micromechanics and microscopy. Indeed, microstructural heterogeneities play a critical role on the macroscopic physical properties of materials. One common way to account for this underlying complexity is resorting to homogenization techniques. Many approaches, including analytical and computational ones, are available for determining the homogenized properties of random media. Most of them necessitate the existence of a representative volume element (RVE). More refined definitions have been given for the RVE over the past 50 years, mostly within the context of micromechanics of elastic media. A review of this topic can be found in Gitman et al. (2007) and Dirrenberger et al. (2014). The clas-

Correspondence to: J. Dirrenberger, PIMM, Arts et Métiers-ParisTech/CNAM/CNRS UMR 8006, 151 bd de l'Hôpital, 75013 Paris, France. Tel: +33 144246101; e-mail: justin.dirrenberger@ensam.eu sical definition of RVE is attributed to Hill (1963), who stated that for a given material the RVE is a sample that is structurally typical of the whole microstructure, i.e. containing a sufficient number of heterogeneities for the macroscopic properties to be independent of the boundary values of traction and displacement. Later, Beran (1968) emphasized the role of statistical homogeneity, especially in a volume-averaged sense. From numerical simulations on volume elements of various sizes, Terada (2000) concluded that from a practical viewpoint, the RVE should be as large as technically possible. Drugan \& Willis (1996) explicitly introduced the idea of minimizing the RVE size, meaning that the RVE would be the smallest material volume for which the apparent and effective properties coincide. It is worth noticing that for a given material the RVE size for morphological or thermal properties is a priori different from the RVE size for elastic properties. Thus, one has to consider an RVE that depends on the specific investigated property. Taking into account these definitions, and assuming ergodicity for the considered heterogeneous media, Kanit et al. (2003) proposed a method based on a statistical analysis for computing the minimal RVE size for a given physical property $Z(\boldsymbol{x}), \forall \boldsymbol{x} \in V$ and precision in the estimate of effective properties. The computed RVE size was found to be proportional to the integral range (Matheron, 1971), which corresponds to a volume of statistical correlation. For a volume $V$ larger than the integral range, $Z$ is considered as a noncorrelated random variable. This approach was implemented in many papers in order to estimate RVE sizes for morphological, elastic and thermal properties, usually resorting to finite element simulations on periodic unit cells of increasing size (Kanit et al., 2003; Jean et al., 2011). The rate of convergence of the mean value for apparent properties, with respect to the size of the considered system, is related to the size of the statistical RVE; i.e. a microstructure with slow rate of convergence would yield large RVE sizes.

Although most of the previous works were related to micromechanics of composite materials, our study deals with the morphology of polymer blends in order to optimize 
functional properties, e.g. permeability, optical refraction, etc. In particular, we focus on nanolayered films, in which two different polymers are combined in a nanostratified structure, composed of numerous alternating thin layers. The nanolayer coextrusion is a continuous process capable of producing films at a large scale with up to thousands of layers, thus yielding individual layer thickness down to several nanometres. Originally developed in the 1960s by Dow Chemical, USA (US Patent No. 3239197; Tollar, 1966), this process was thoroughly investigated by Baer's group to study nanoscale polymer interactions (Liu et al., 2003) and produce films with unique optical properties (Kazmierczak et al., 2007), as well as enhanced mechanical (Kerns et al., 1999) or gas barrier properties (Wang et al., 2009). In our laboratory, the process has been recently used to control the architecture at the micro-/nanoscale of multiphase polymer systems, like polymer blends (Boufarguine et al., 2013), nanocomposites (Miquelard-Garnier et al., 2013; Li et al., 2014) or triblock copolymers (Roland et al., 2016).

Since multilayered coextrusion results in materials exhibiting more or less regular microstructures, the development of a characterization method enabling a full description of the morphological features of multilayered systems is of prime importance. When the number of layers is too large for individual characterization, resorting to statistical approaches becomes mandatory in order to account for the behaviour of the whole material. In this case, a large density of microstructural heterogeneities complies with the requirements of classical RVE definitions, hence enabling the correlation between microstructural features and macroscopic performance. This approach is desirable when optimizing the process and investigating the effect of process parameters on the layer thickness distribution. As a matter of fact, the RVE size will depend on such process parameters, for the statistical rate of convergence of apparent properties is intrinsically related to the microstructural variability induced by the process.

In this work, the layer heterogeneities are characterized by very small, nanometric, length scales, which makes their observation difficult. Appropriate methods, such as atomic force microscopy (AFM) or transmission electron microscopy, are available, but with reduced regions of interest under observation, of a few squared microns at best, hence bringing back the question of representativity for the micrographs acquired this way. This practical problem of scale separation and representativity is very similar to what has been encountered by other authors in the literature when trying to evaluate RVE sizes on various materials, such as fibrous media (Dirrenberger et al., 2014), collagen fibrils (Altendorf et al., 2012), concrete (Huet, 1990; Pelissou et al., 2009) or particle-reinforced composites (Salmi et al., 2012).

In order to tackle the problem of sample representativity, the statistical method proposed by Kanit et al. (2003), although initially developed by Hersant \& Jeulin (1976), for determining RVE sizes will be implemented for morphological properties of nanolayered polymer films, i.e. the layer thickness $t$ and volume fraction $V_{V}$, based on AFM micrographs.

First, the material of interest, as well as the characterization techniques used for sample observation, is presented. The threshold and micrograph treatment are then introduced as well as the image analysis procedure, which was used to morphologically describe the samples. Sample population statistics are then used as inputs for estimating the representative sample size depending on process parameters. Finally, results on representativity are used to discriminate potential bias in observation techniques.

\section{Materials and characterization techniques}

\section{Materials}

Nanolayered polymer PS-PMMA films were considered in this work. Poly(methyl methacrylate) (PMMA) was graciously supplied by Altuglas International (Altuglas VM100), whereas polystyrene (PS) was provided by Total Petrochemical (Crystal 1340). The melt flow indexes (MFIs), densities and complex viscosity $\eta^{*}$ in the extrusion conditions $\left(225^{\circ} \mathrm{C}-\dot{\gamma}=5 \mathrm{~s}^{-1}\right)$, determined using an Anton Paar rheometer in plate/plate configuration, are given in Table 1.

\section{Process}

PS-PMMA nanolayered films are manufactured using a multilayer coextrusion process. The processing route consists of two 20-mm single-screw extruders with melt gear pumps, a three-layer coextrusion feed-block (A-B-A), a series of layermultiplying elements, an exit flat die and a thermally regulated chill roll as illustrated in Figure 1. PMMA was extruded to form the outer skin layers and PS the core layer. The inclusion of gear pumps into the coextrusion system enables an additional degree of control over the relative thickness ratio of the layered polymers as they enter the A-B-A feed-block. In this study, the mass fraction of polymer B in the film was set and kept constant by adjusting the flow rate through the speed of the melt gear pumps. The initial three-layer polymer flow subsequently enters a mixing section, composed of a sequence of layer-multiplying elements. The melt was initially cut in half vertically, and then each half was compressed and restretched to its original width, hence doubling the number of layers with each layer-multiplying element. A series of $n$ elements combines two polymers producing $2^{n+1}+1$ alternating layers, as shown in Figure 1.

Here, 10 multiplying elements were used, giving films containing 2049 layers. Finally, after passing through the last layer-multiplying element, the nanolayered structure was formed into a thin sheet by passing through a flat die, 150 $\mathrm{mm}$ wide and $1.5 \mathrm{~mm}$ thick. At the die exit, the nanolayered samples were stretched and quenched, using a water-cooled chill roll at $95^{\circ} \mathrm{C}$, and collected. The resulting sample is a 
Table 1. Rheological properties of the polymers used in this work.

\begin{tabular}{llclc}
\hline Polymer & Commercial name & Density $\left(\mathrm{g} / \mathrm{cm}^{3}\right)^{(1)}$ & $\mathrm{MFI}^{(1)}$ & $\eta^{*}$ at $225^{\circ} \mathrm{C}-\dot{\gamma}=5 \mathrm{~s}^{-1}$ \\
\hline PMMA & Altuglas VM100 & 1.18 & $14.5 \mathrm{~g} / 10 \mathrm{~min}$ at $230^{\circ} \mathrm{C} / 3.8 \mathrm{~kg}$ & $791 \mathrm{~Pa} . \mathrm{s}$ \\
PS & Crystal 1340 & 1.05 & $4.0 \mathrm{~g} / 10 \mathrm{~min}$ at $200^{\circ} \mathrm{C} / 5 \mathrm{~kg}$ & $786 \mathrm{~Pa} . \mathrm{s}$ \\
\hline
\end{tabular}

${ }^{(1)}$ Obtained from technical datasheet.

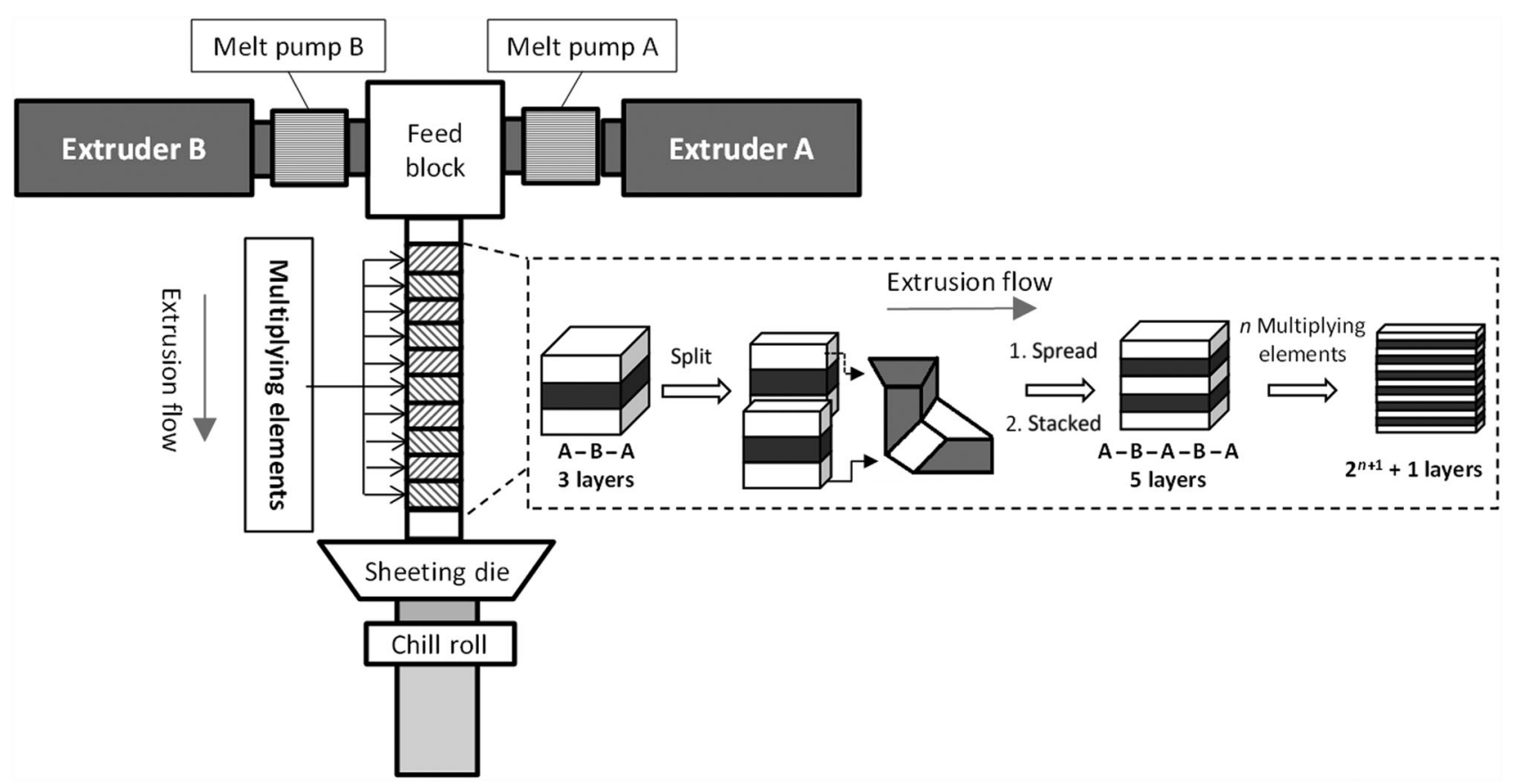

Fig. 1. Principle of the multiplication of layer by the multilayer coextrusion process to fabricate the films.

rectangular film made of alternating layers of PS and PMMA, architectured as a one-dimensional (1D) stacking. The sample has PS/PMMA compositions by weight of 10/90 and a thickness of approximately $250 \mu \mathrm{m}$. Based on these parameters, the nominal PS layer thickness is $27 \mathrm{~nm}$ (Eq. 1) and the theoretical volume fraction is $11 \%$.

$$
\begin{aligned}
& \text { Nominal thicknesses } \\
& =\text { Thickness }_{\text {film }} \times \frac{\text { Vol } \% \text { PS }}{\text { Number of PS layers }}
\end{aligned}
$$

\section{Characterization techniques}

AFM images were obtained in tapping mode using a multimode microscope controlled by a Nanoscope V controller (Veeco), operated under ambient atmosphere. The tips (silicon, spring constant $40 \mathrm{~N} / \mathrm{m}$, oscillation frequency ca. $300 \mathrm{kHz}$ ) were obtained from BudgetSensors. The radius of curvature of the tips was less than $10 \mathrm{~nm}$. Phase, height and amplitude images were acquired simultaneously. Specimens were cut from the centre of the extruded films and sectioned perpendicular to their surface with an ultramicrotome 2088 Ultrotome V (LKB) at a cutting speed of $1 \mathrm{~mm} / \mathrm{s}$. Images were recorded at full resolution $(4096 \times 4096$ pixels), with a scan rate of $0.5 \mathrm{~Hz}$. This resolution yields a pixel size of $7 \mathrm{~nm}$. AFM images were taken from extrusion direction (Fig. 2). The phase signal was described as a measure of the energy dissipation involved in the contact between the tip and the sample, which depends on a number of factors, including viscoelasticity, adhesion and topography. As these factors are different between PS and PMMA, the thickness of layers was measured from the AFM phase images (Fig. 2), which most clearly revealed the layered film structure. On the obtained images, PS and PMMA appear in brown and gold colour, respectively.

\section{Image analysis}

Since the film has finite dimensions, 10 images of around 100 layers were taken all along the thickness of the film as represented in Figure 2. The sample was composed of 1024 PS layers; a large fraction of PS layers (ca. 80\%) was measured indeed. In order to determine the RVE, these 10 images were divided into nonoverlapping, neighbour squares of equal size. Thus, due to the regular nature of the microstructure, it was possible to obtain statistical data for more than 100 layers by 


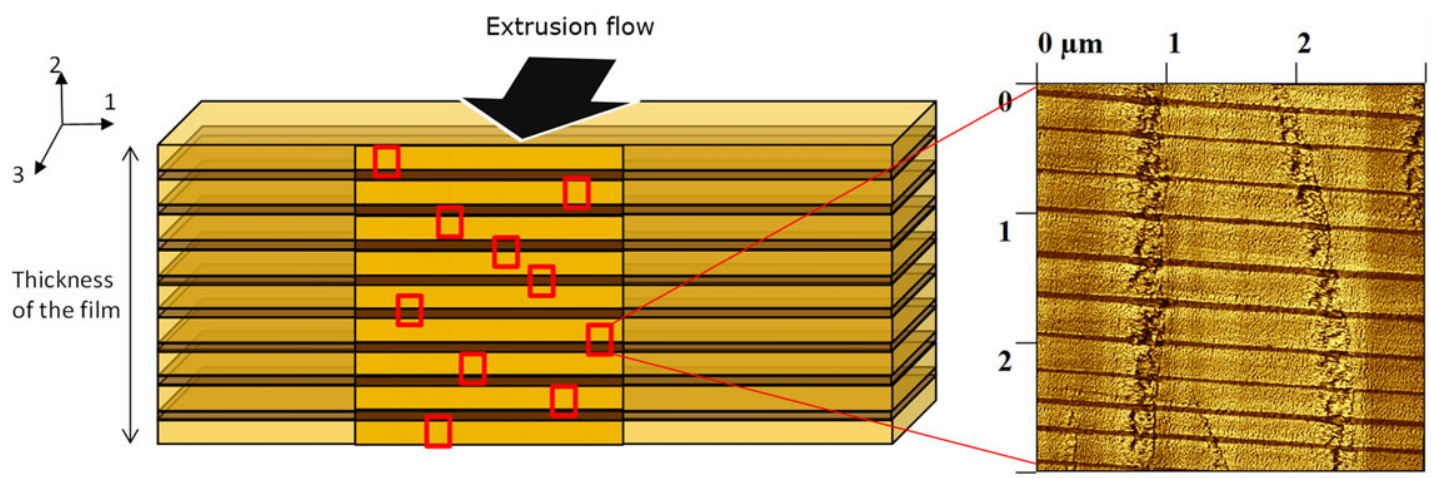

Fig. 2. AFM specimen and image analysis principle. The arrow represents the extrusion flow direction (left); AFM image of partial cross section of the sample (vertical lines are compression lines due to sample preparation, right).
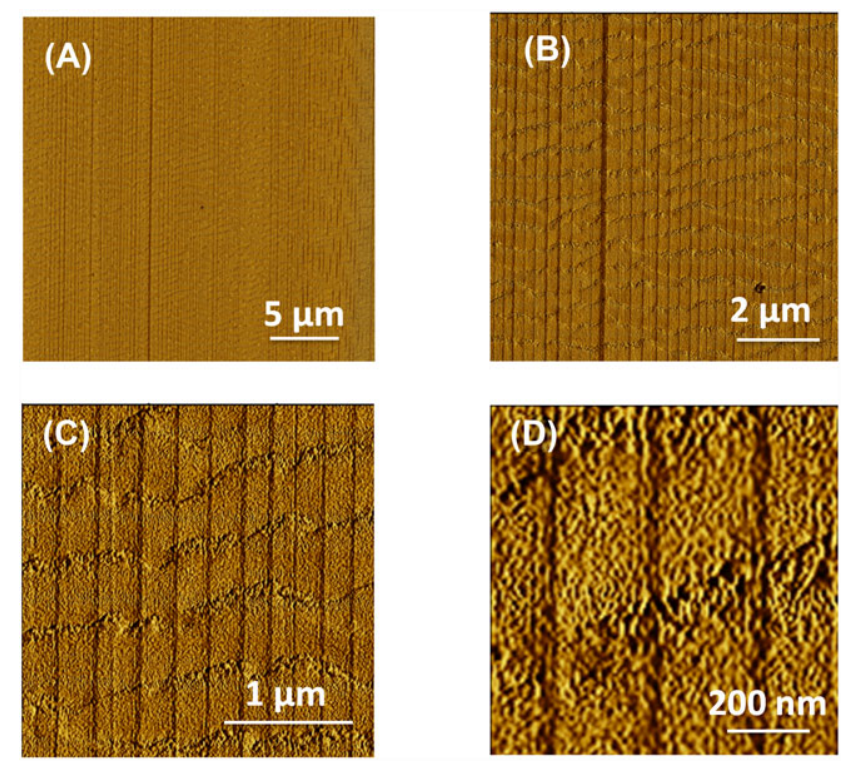

Fig. 3. AFM phase images of PMMA/PS (90/10 wt\%) film with (A) 100 layers, (B) 30 layers, (C) 10 layers and (D) 3 layers.

compiling them. In the end, seven series of images containing respectively 3,10, 30, 100, 200, 300 and 500 layers of PS were obtained, as shown in Figure 3, for the first four series. The horizontal lines on these AFM images were chatter marks due to sample compression during microtoming. The characteristics for each series are given in Table 2. The number of realizations corresponds to the number of samples considered within one series.

Thicknesses of PS layers were measured from AFM phase images with the image analysis software Gwyddion. Through the software, a phase profile can be extracted showing the variation of phase degree, as shown in Figure 4. It is noteworthy that the profile is averaged over 128 pixels whatever be the image size. This integrated height is larger than the thickness of the cut and compression lines, which appear during the sample preparation. Moreover, these lines are perpendicular to the layers. Hence, they are included in the profile noise, and the measurements of layer thickness are not affected. Each layer is represented by one peak on the profile. The thickness of each layer is determined according to an arbitrary procedure which consists of measuring the full width at half-maximum height of the peak. This step is similar to a manual threshold.

As the thickness of layers is in the range of tens of nanometres, i.e. a few pixels in terms of AFM imaging, it is critical to analyse all possible sources of error. Various types of error can exist in this case: uncertainties of measurement, systematic error, and sampling error. The size of the AFM tip, AFM controller, image compression and acquisition definition were considered as uncertainties of measurement. The manual threshold and bias due to the operator were considered as systematic error. The sampling, which depends on the size of the considered system, i.e. the total number of layers which will be measured, can be a source of error if the number of analysed layers is too small. This last point is fundamental for our study as the sampling error can be related to the RVE size, given the assumptions of statistical homogeneity and ergodicity for the considered material. Both assumptions will be made from now on in order to provide a consistent ground for applying the approach developed by Kanit et al. (2003).

Tips used have a curvature radius of $R=10 \mathrm{~nm}$. The inplane resolution of AFM is related to the radius of curvature of the tip, as well as the vertical detection limit $(\Delta z=0.1 \mathrm{~nm}$ in our case, given by the manufacturer), and the size of the feature being characterized. The in-plane detection limit is approximated by Eq. (2), as described in Appendix A.

$$
\Delta x=2 \sqrt{2 R \Delta z}
$$

The value estimated from Eq. (2) is $\Delta x=2.8 \mathrm{~nm}$, which is considerably small in comparison with the theoretical value of PS layers $(27 \mathrm{~nm})$. Therefore, the uncertainty of measurement due to the AFM tip size was considered negligible. To reinforce this assertion, a comparative study has been done with a thinner tip $(R=2 \mathrm{~nm})$ and results regarding the layer thickness were the same. All images were acquired by the same 
Table 2. Characteristics of each volume series.

\begin{tabular}{lccccccc}
\hline & 3 layers & 10 layers & 30 layers & 100 layers & 200 layers & 300 layers & 500 layers \\
\hline Number of realizations & 275 & 81 & 28 & 10 & 5 & 3 \\
Average sample size (nm) & 738 & 2441 & 6949 & 20143 & 40287 & 60076 \\
Total number of PS layers & 825 & 810 & 840 & 1000 & 1000 & 900717 \\
Number of measured PS layers & 822 & 808 & 800 & 822 & 822 & 742 \\
\hline
\end{tabular}

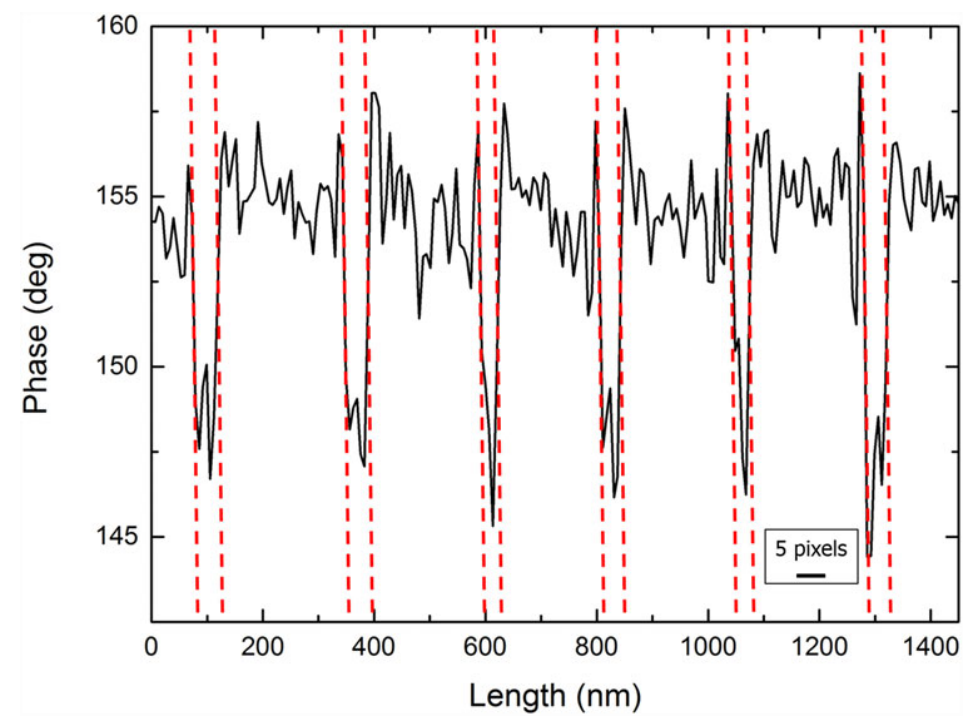

Fig. 4. Example of phase profile in Gwyddion.

operator with the same AFM controller at a constant image resolution $(4096 \times 4096$ pixels at a scan rate of $0.5 \mathrm{~Hz})$ in order to avoid image resolution bias. However, it is worth noting that the images were acquired with the highest resolution attainable for this AFM apparatus. The acquisition definition can have a crucial importance on measurements if not chosen cautiously. The minimal acceptable resolution can be defined by a criterion, e.g. that one pixel represents less than $10 \%$ of the measured feature. AFM images are recorded as raw data, without algorithmic compression, hence no error due to image file compression was considered. Concerning threshold, with this measurement method, the systematic error on thickness was estimated to one pixel. The size of the pixel depends on the size of the image and the resolution. Here, the systematic error was $27000 / 4096$, being $7 \mathrm{~nm}$. Moreover, this measurement method overestimates the value by including the external pixels in the measure, as shown in Figure 4. So, for each value measured on the profile, the value of systematic error was subtracted in order to improve accuracy.

\section{Representativity of samples}

The physical interpretation of the integral range is such that for a given volume $V$, one can define $n=V / A_{3}$ volume elements for which the $i$ averaged values $Z_{i}\left(V^{\prime}\right)$ over the $n$ subvolumes
$V^{\prime}=V / n$ are uncorrelated random variables. For a large specimen, i.e. $V \gg A_{3}$, the ensemble variance $D_{Z}^{2}(V)$ can then be expressed introducing the point variance of the physical field $Z(\boldsymbol{x}), D_{Z}^{2}$ as follows (Matheron, 1971; Matheron, 1989):

$$
D_{Z}^{2}(V)=D_{Z}^{2} \frac{A_{3}}{V}
$$

with $A_{3}$, the integral range in $R^{3}$ defined as :

$$
A_{3}=\frac{1}{D_{Z}^{2}} \int_{R^{3}} \overline{W_{2}}(h) d h
$$

$\overline{W_{2}}(h)$ is the centred second-order correlation function such that, for properties $Z_{1}$ and $Z_{2}$, respectively within the PS and PMMA phases, and probability $p$ for $x \in V_{1}$, i.e. the volume of PS phase:

$$
\begin{aligned}
\overline{W_{2}}(h) & =\overline{(Z(\boldsymbol{x}+\boldsymbol{h})-\bar{Z})(Z(\boldsymbol{x})-\bar{Z})} \\
& =\left(Z_{1}-Z_{2}\right)^{2}\left(C(h)-p^{2}\right)
\end{aligned}
$$

With $C(h)$ the morphological covariance of a random closed set $A$ such that:

$$
C(\boldsymbol{x}, \boldsymbol{x}+\boldsymbol{h})=P\{\boldsymbol{x} \in A, \boldsymbol{x}+\boldsymbol{h} \in A\}
$$


with $\boldsymbol{x}$ the material point vector and $\boldsymbol{h}$ an arbitrary vector of modulus $h$.

In the case of concern for this work, i.e. nanolayered polymer blends ideally structured as a 1D stacking, morphological variability is induced in only one direction, the microstructural morphology being constant in both directions 2 and 3. Equations (3) and (4) can thus be reformulated as Eqs. (7) and (8) by considering the sample size $L$, or length of the sample, e.g. in $\mu \mathrm{m}$ :

$$
D_{Z}^{2}(L)=D_{Z}^{2} \frac{A_{1}}{L}
$$

with $A_{1}$, the integral range in $R$ defined as :

$$
A_{1}=\frac{1}{D_{Z}^{2}} \int_{R} \overline{W_{2}}(h) d h
$$

Following the method proposed by Matheron (1989) and implemented by Kanit et al. (2003) considering a large number $n$ of realizations (or subvolumes), the following absolute sampling error $\epsilon_{a b s}$ in the estimation of the effective properties arises:

$$
\epsilon_{a b s}=\frac{2 D_{Z}(L)}{\sqrt{n}}
$$

From which the relative error $\epsilon_{\text {rel }}$ can be defined:

$$
\epsilon_{r e l}=\frac{\epsilon_{a b s}}{\bar{Z}}=\frac{2 D_{Z}(L)}{\bar{Z} \sqrt{n}} \rightarrow \epsilon_{r e l}^{2}=\frac{4 D_{Z}^{2} A_{1}}{\bar{Z}^{2} n L}
$$

Hence, the following sample size can be considered as statistically representative for a prescribed property $Z$, number of realizations $n$ and relative error:

$$
L_{R V E}=\frac{4 D_{Z}^{2} A_{1}}{\epsilon_{r e l}^{2} \bar{Z}^{2} n}
$$

This RVE size then depends on the point variance $D_{Z}^{2}$, integral range $A_{1}$ and mean value $\bar{Z}$. These three parameters are estimated from the image analytically, except when considering the volume fraction which is equal to the length fraction $L_{L}$, for which $D_{Z}^{2}$ is known explicitly:

$$
D_{L_{L}}^{2}=L_{L}\left(1-L_{L}\right)
$$

For the specific case of PS layer thickness, the theoretical thickness can be obtained from the length fraction as follows:

$$
t_{t h}=L_{L} \frac{L}{N}
$$

with $L$, size of the sample and $N$, the number of PS layers within the sample, which is a constant imposed by the number of multiplying elements used during the coextrusion process and the sample size L. Hence, Eq. (12) can be adapted in the following way for the point variance of PS layer thickness:

$$
D_{t}^{2}=\left(\frac{L}{N}\right)^{2} L_{L}\left(1-L_{L}\right)
$$

Table 3. Relative sampling error $\left(\epsilon_{\text {rel }}\right)$ associated with the morphological properties and size of the system.

\begin{tabular}{lccc}
\hline Sample size & $\begin{array}{c}\text { Number of } \\
\text { realizations }\end{array}$ & $\begin{array}{c}\epsilon_{\text {rel }}-\text { PS layer } \\
\text { thickness }\end{array}$ & $\begin{array}{c}\epsilon_{\text {rel }}-\text { PS volume } \\
\text { fraction }\end{array}$ \\
\hline 3 layers & 275 & $4.9 \%$ & $4.4 \%$ \\
10 layers & 81 & $5.9 \%$ & $5.2 \%$ \\
30 layers & 28 & $7.2 \%$ & $5.9 \%$ \\
100 layers & 10 & $7.7 \%$ & $4.2 \%$ \\
200 layers & 5 & $2.6 \%$ & $4.3 \%$ \\
300 layers & 3 & $5.1 \%$ & $0.7 \%$ \\
500 layers & 2 & $0.4 \%$ & $0.3 \%$ \\
\hline
\end{tabular}

It was proposed by Lantuéjoul (1991) to use a modified scaling law with the exponent $\gamma \neq 1$ for situations in which the hypothesis of an ergodic stationary random function with finite integral range is not fulfilled. The exponent $\gamma$ can be deduced theoretically in the case of Boolean random varieties with nonfinite integral range (Jeulin, 2015), or estimated heuristically from the statistical treatment of image analyses in the case of morphological properties, or computations for any simulated physical property. The variance can thus be rewritten as follows (Jeulin, 2011):

$$
D_{Z}^{2}(L)=D_{Z}^{2}\left(\frac{A_{1}^{*}}{L}\right)^{\gamma}
$$

where $A_{1}^{*}$ is not the integral of the centred second-order correlation function $\overline{W_{2}}(h)$ anymore. Nonetheless, it is equivalent to a length of material and can readily be used to determine RVE sizes which can then be obtained by updating the previous definition of the relative error:

$$
\epsilon_{r e l}=\frac{\epsilon_{a b s}}{\bar{Z}}=\frac{2 D_{Z}(L)}{\bar{Z} \sqrt{n}} \rightarrow \epsilon_{r e l}^{2}=\frac{4 D_{Z}^{2} A_{1}^{* \gamma}}{\bar{Z}^{2} n L^{\gamma}}
$$

Hence, yielding an updated definition of the RVE size:

$$
L_{R V E}=A_{1}^{*}\left(\frac{4 D_{Z}^{2}}{\epsilon_{r e l}^{2} \bar{Z}^{2} n}\right)^{\frac{1}{\gamma}}
$$

Using Eq. (16), the relative error associated with the number of considered samples is computed and presented hereafter in Table 3. Ideally, the error should be the same for all sample series by adapting the population. Although the relative error fluctuates, most values are very low, with a maximum value below $8 \%$ on the mean PS layer thickness. The coefficient $A_{1}^{*}$ and scaling-law exponent $\gamma$ can be estimated from image analysis as it was done by Kanit et al. (2003), Altendorf et al. (2014) and Wang et al. (2015), by considering the ensemble variance $D_{Z}^{2}(L)$ versus $L$ and identifying the ordinate at the origin for the scaling law, hence yielding $D_{Z}^{2} A_{1}^{* \gamma}$ from which exponent $\gamma$ and point variance $D_{Z}^{2}$ are known, leaving only $A_{1}^{*}$ to be evaluated.

When considering statistical RVE sizes of microstructures with no information about the integral range and theoretical 

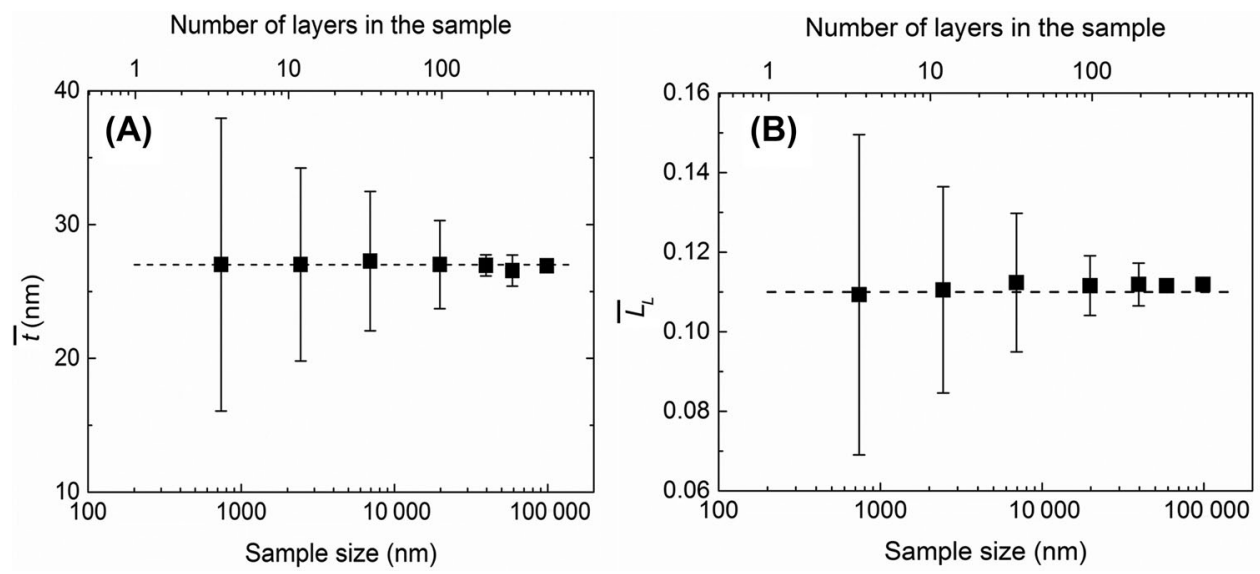

Fig. 5. Mean values for the PS layer thickness $t(\mathrm{~A})$ and volume fraction $L_{L}(\mathrm{~B})$ varying with sample size.
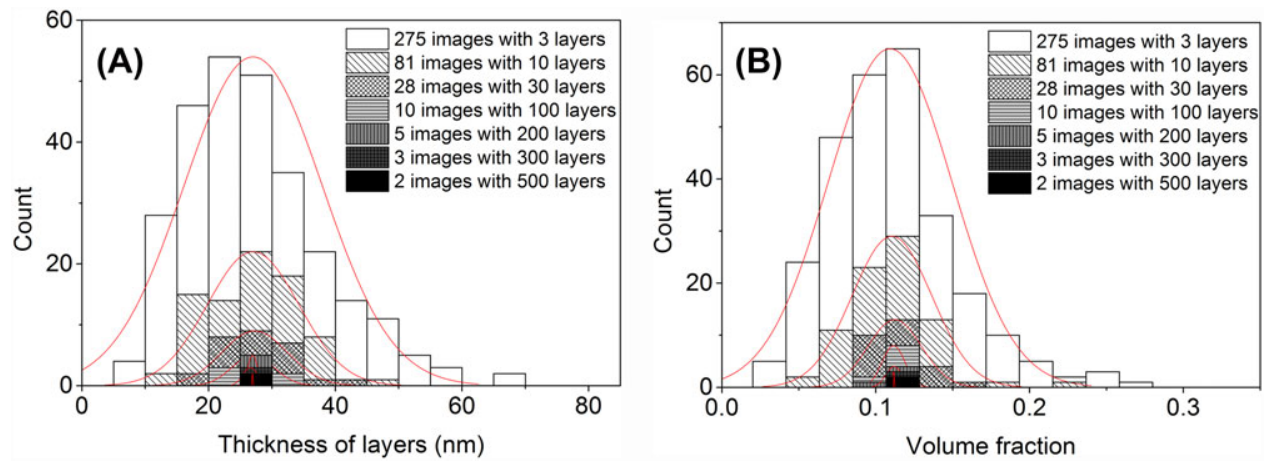

Fig. 6. Distribution of statistical population as a function of (A) thickness of layers and (B) volume fraction. The red lines represent the normal distribution curves.
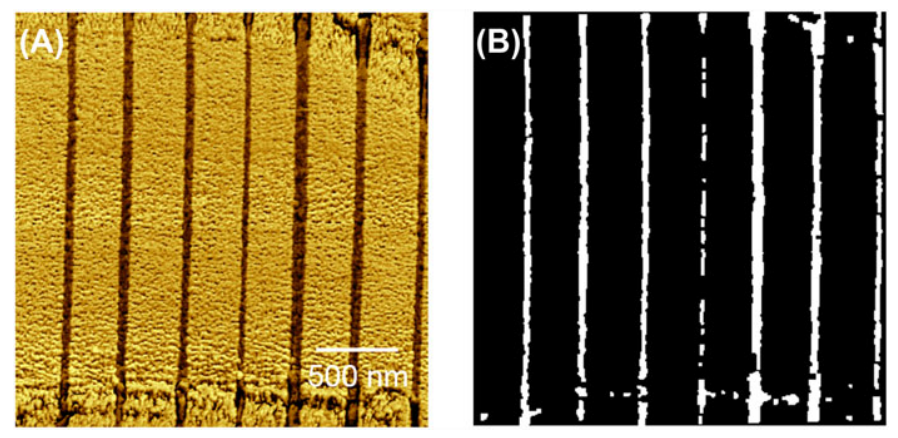

Fig. 7. (A) AFM micrograph used for the covariance study and (B) binary image for computing the covariance.

value of the point variance $D_{Z}^{2}$, it may be useful to reformulate Eq. (15) as follows:

$$
D_{Z}^{2}(L)=K L^{-\gamma}
$$

with $K=D_{Z}^{2} A_{1}^{* \gamma}$, leaving only two parameters to be identified from the statistical analysis. Equation (17) can thus be updated in this way:

$$
L_{R V E}=\left(\frac{4 K}{\epsilon_{r e l}^{2} \bar{Z}^{2} n}\right)^{\frac{1}{\gamma}}
$$

\section{Results and discussion}

Morphological measurements have been performed on the six different populations of sample size.

\section{Mean value and distribution}

The mean volume fraction of PS layers $V_{V}$ is equal to both the surface fraction $S_{S}$ and length fraction $L_{L}$ of PS, since the microstructural morphology is stationary. $\overline{L_{L}}$ has been computed over $n$ realizations for a given sample size $L$ in nm. 


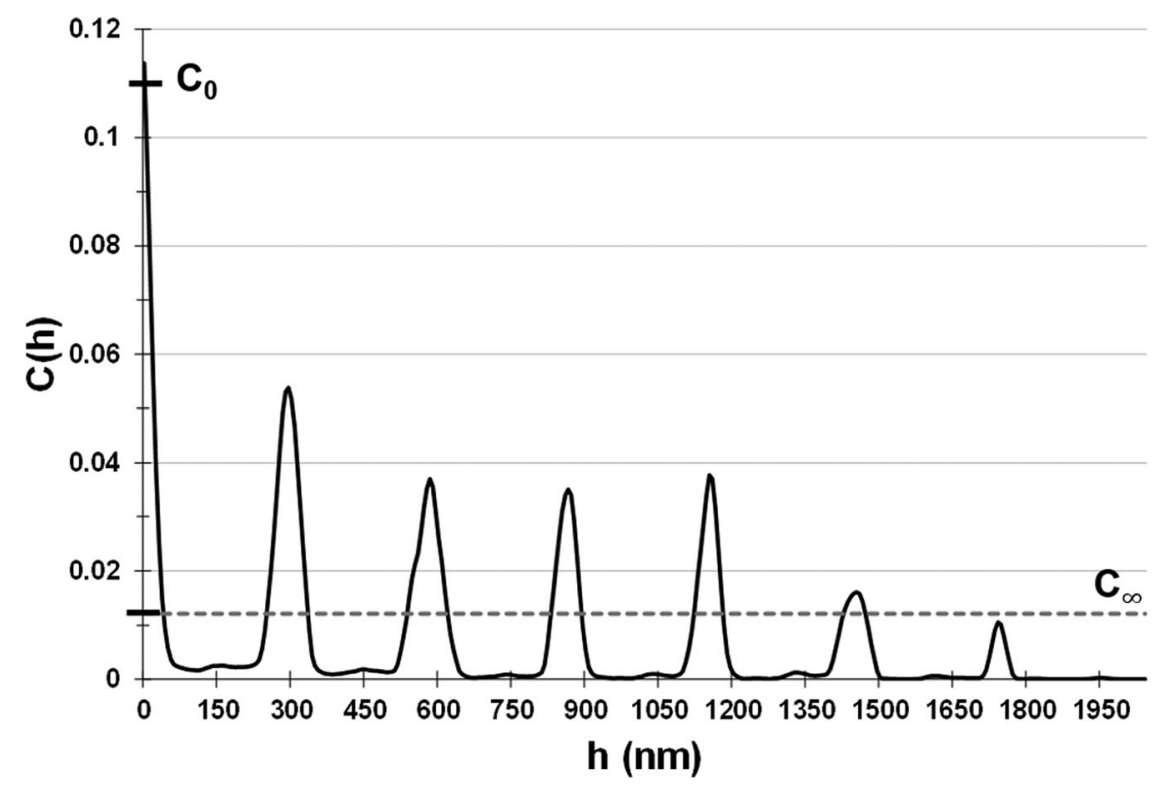

Fig. 8. Covariance computed horizontally on the image shown in Figure 7(B).

The mean PS layer thickness $\bar{t}$ was also estimated from image analysis with respect to the size of the sample. Both $\overline{L_{L}}$ and $\bar{t}$ are plotted as functions of the sample size in Figure 5. The mean values obtained for the largest sample size considered $(L=98862 \mathrm{~nm})$ are $\overline{L_{L}}=11.19 \pm 0.03 \%$ for the volume fraction and $\bar{t}=26.9 \pm 0.1 \mathrm{~nm}$. Morphological fluctuations are inherent to the stochastic nature of real-life materials. As expected, no bias occurs for both the layer thickness and volume fraction, whatever the size of the realizations.

The observed distributions of thickness and volume fraction of PS layers for each sample size $L$ are shown in Figures 6(A) and (B), respectively. For each property, the distributions were similar whatever be the considered system. As represented in red lines in Figure 6, normal distribution curves have been fitted to the experimental data. Experimental data and fit are in good agreement. For each population, the mean value of the normal distribution curve was equal to $\bar{t}$. This result confirms the implicit hypothesis of standard deviation calculation made with Eq. (10) from which the statistical analysis is done.

\section{Covariance}

In order to check for morphological regularity of the material considered, the two-point geometrical covariance was computed for the sample shown in Figure 7(A), which was transformed into the binary image (Fig. 7B) by manual thresholding and morphological opening and closure operations. The considered sample was approximately $2 \times 2 \mu \mathrm{m}$, including seven PS layers and a volume fraction of PS layers of $11.4 \%$. Its covariance was estimated along the horizontal direction, the orientation of vector $\boldsymbol{h}$, which corresponds to direction 2

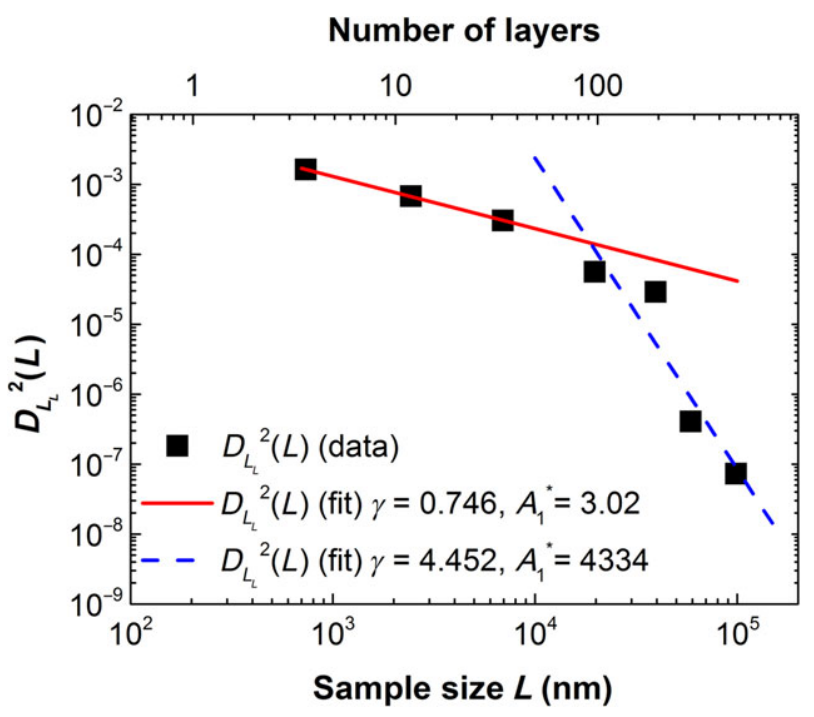

Fig. 9. Variance $\boldsymbol{D}_{\boldsymbol{L}_{\boldsymbol{L}}}{ }^{2}(\boldsymbol{L})$ of the volume fraction of PS depending on sample size $\boldsymbol{L}$, computed from image analysis.

in Figure 2. The regular quasiperiodic character of the material is clearly apparent on the covariance plot shown in Figure 8 , yielding a distance of $280 \mathrm{~nm}$ between the centres of two neighbouring PS layers. The first point $\mathbf{C}_{0}$ corresponds to the volume fraction of the sample: $V_{V}=0.114$, whereas the sill $\mathrm{C}_{\infty}$ corresponds to $V_{V}^{2}=0.013$.

\section{Morphological representativity}

Results regarding the volume fraction are presented in Figure 9. Variance for the mean PS layer thickness as a 


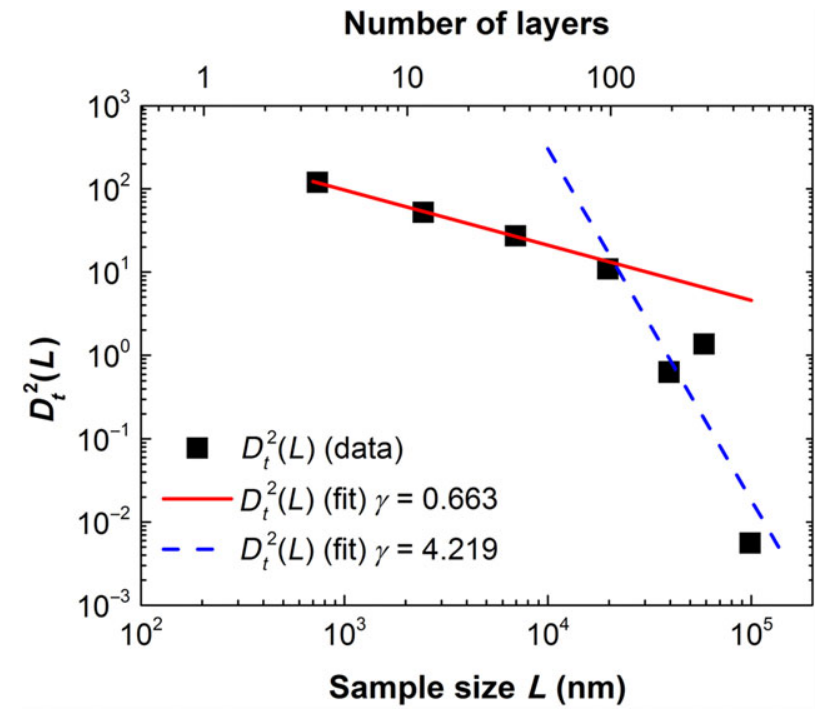

Fig. 10. Variance $\boldsymbol{D}_{t}^{2}(\boldsymbol{L})$ of the thickness of PS layers depending on the sample size $\boldsymbol{L}$, computed from image analysis.

function of the sample size is shown in Figure 10. The $\gamma$ exponents of the scaling law for each morphological property were estimated from the results of image analysis, by fitting the slope of the variance curves. Values of $K$ are estimated from Figures 9 and 10 based on Eq. (18). From Figure 9, two slopes are identified for the power law, indicating the existence of two scales of heterogeneities. The first-scale, or local, variability is intrinsic to the microstructure induced by the extrusion process: it encompasses the effects of short-range physical phenomena, such as flow nonlinearities, local thermal inhomogeneity and interfacial interactions. This first scale of variability is always present although its effects become blunter for a larger system; it is characterized by a consistent $\gamma$ exponent of $0.66-0.75$ for both properties, which should be compared to the theoretical value of 0.5 obtained for random fibres in 2D (Jeulin, 2015). The second scale of variability to consider is seen only for sample sizes higher than $10^{4} \mathrm{~nm}$; its origin could be described as boundary effect patterns during the process. Indeed, due to higher shear rate prescribed to the melt at the wall while passing through multiplying elements, layers in the vicinity of the wall become thinner than others. If this phenomenon occurs at each multiplying step, the final sample is constituted of patterns with long-range varying layer thickness sequences. The tipping point between the slopes could then be interpreted as the characteristic length of such pattern. In our case, the pattern dimension can be estimated to be $2.10^{4} \mathrm{~nm}$, corresponding to approximately 100 layers, i.e. about $10 \%$ of the film thickness. Rather than considering this a limitation of the statistical approach invoked for the case of films with finite dimensions, we propose to use this method for the characterization of microstructural variability, in order to study the effect of process parameters on the quality of nanolayered films. As a matter of fact, this statistical approach allows for the discrimination of multiple sources of variability and interpretation of their physical meaning. The second scale of variability appears to accelerate the statistical convergence with respect to the size of the system, for volume fraction $(\gamma=4.45)$, as well as for the mean layer thickness $(\gamma=4.22)$. Also such exponents are expected for random media with zero integral range, which is the case for the periodic part of the microstructure, as revealed by the quasiperiodic behaviour of the covariance in Figure 8. Nevertheless, in this study, sample series containing 100 layers or more have a low number of realizations $(\leq 10)$. More samples would be necessary in order to obtain a better accuracy for these series.

Using Eq. (19), it is now possible to determine statistical RVE sizes from image analysis. Estimates for RVE sizes are presented in Table 4 for the different morphological properties (volume fraction and layer thickness of PS), for various relative errors and numbers of realizations ( $n=\{1 ; 10 ; 50\})$, using the first-scale variability parameters, since only small size samples are readily accessible with AFM. RVE sizes presented in this table for $n=1$ are always larger than the volume element sizes achieved throughout this work: $L=100 \mu \mathrm{m}$ on average for the largest. Nevertheless, the precision for a given sample size can be obtained from multiple realizations of smaller samples. As an example, for $\bar{t}_{P S}$, if $L=12 \mu \mathrm{m}$, and $\epsilon_{\text {rel }}=10 \%$, one must analyze 10 realizations in order to obtain the same statistical convergence as for one realization with $L=400 \mu \mathrm{m}$. Precaution should be taken regarding the bias induced by boundary layer effects on mean values by choosing smaller elementary samples when considering physical properties rather than morphological ones.

Finally, values for RVE size and relative error in Table 4 can be compared to the sample sizes considered and associated relative errors in Table 3; for instance, if one considers the experimental error associated with the estimate of $L_{L}$ for $N=$ 10 layers, i.e. $L=2455 \mathrm{~nm}$ with $n=81$ realizations, $\epsilon_{\text {rel }}=$ $5.2 \%$. For the same relative error and number of realizations, the asymptotic model, on which values in Table 4 are based, yields an RVE size of $L=2470 \mathrm{~nm}$, which corresponds to an error of $1 \%$ in terms of sample size prediction for a given precision. The model seems appropriate for the considered data range.

From both practical and acquisition time viewpoints, for mean value and distribution of layer thickness for a given precision, it is better to analyse images with fewer layers. So, the power-law parameters to be considered for the determination of a representative sample size are those related to the firstscale variability. In order to characterize long-range boundary effects due to the manufacturing process, large samples have to be considered. Such image acquisition might be inaccessible through AFM, and therefore large samples can be reconstructed from smaller contiguous nonoverlapping samples, as it was done in this work. 
Table 4. $\boldsymbol{L}_{\boldsymbol{R} \boldsymbol{V} \boldsymbol{E}}$ sizes estimated using Eq. (19) for PS volume fraction and layer thickness.

\begin{tabular}{|c|c|c|c|c|c|c|}
\hline Z & $\gamma$ & K & $n$ & $\epsilon_{\text {rel }}$ & $L_{R V E}(\mathrm{~nm})$ & $\bar{N}$ \\
\hline \multirow[t]{5}{*}{ PS volume fraction } & 0.746 & $7.0810^{-2}$ & 1 & $5 \%$ & $9.9210^{5}$ & 4043 \\
\hline & 0.746 & $7.0810^{-2}$ & 10 & $5 \%$ & $4.5310^{4}$ & 185 \\
\hline & 0.746 & $7.0810^{-2}$ & 1 & $10 \%$ & $1.5510^{5}$ & 630 \\
\hline & 0.746 & $7.0810^{-2}$ & 10 & $10 \%$ & $7.0710^{3}$ & 29 \\
\hline & 0.746 & $7.0810^{-2}$ & 50 & $10 \%$ & $8.1710^{2}$ & 3 \\
\hline & 0.663 & $9.4310^{3}$ & 10 & $5 \%$ & $1.0010^{5}$ & 409 \\
\hline & 0.663 & $9.4310^{3}$ & 50 & $5 \%$ & $8.8510^{3}$ & 36 \\
\hline & 0.663 & $9.4310^{3}$ & 1 & $10 \%$ & $4.0010^{5}$ & 1628 \\
\hline & 0.663 & $9.4310^{3}$ & 10 & $10 \%$ & $1.2410^{4}$ & 51 \\
\hline & 0.663 & $9.4310^{3}$ & 50 & $10 \%$ & $1.0910^{3}$ & 4 \\
\hline
\end{tabular}

\section{Conclusions and perspectives}

- Nanolayered PS-PMMA polymer blend films were manufactured and morphologically characterized through AFM and image analysis. Representativity of hundreds of nanoscale heterogeneous samples was investigated.

- The statistical approach introduced by Kanit et al. (2003) was adapted and implemented for the case of 1D nanolayered materials based on image analys of microstructural samples. RVE sizes were determined for both PS volume fraction and mean layer thickness.

- The study of the ensemble variance convergence with respect to the size of the system revealed two regimes for the scaling power law, indicating the presence of two scales of morphological heterogeneities within the material.

- In summary, three functions are enabled by the present approach:

- To predict RVE size for a given property and precision.

- To reach the same precision with either one large sample or several smaller samples.

- To discriminate and characterize multiple scales of variability in heterogeneous media.

Further work will include the morphological modelling of such materials in order to generate populations of virtual samples for computation of physical properties, e.g. mechanical, thermal, electrical, etc. As the rate of statistical convergence with respect to the size of the system informs us about variations induced in the microstructure, the current approach will be applied for different factors of influence, i.e. blend compositions, morphologies and process parameters. This work is a useful step further towards understanding the relationship between process parameters, induced microstructures and functional properties.

\section{Acknowledgement}

The authors would like to gratefully acknowledge financial support from the French government agency ADEME and Région Aquitaine through the ISOCEL research project.

\section{References}

Altendorf, H., Decencière, E., Jeulin, D., et al. (2012). Imaging and 3D morphological analysis of collagen fibrils. J. Microsc. 247(2), 161-175.

Altendorf, H., Jeulin, D. \& Willot, F. (2014) Influence of the fiber geometry on the macroscopic elastic and thermal properties. Int. J. Solids Struct. 51, 3807-3822.

Beran, M. (1968) Statistical Continuum Theories. Wiley, New York.

Boufarguine, M., Guinault, A., Miquelard-Garnier, G. \& Sollogoub, C. (2013) PLA/PHBV films with improved mechanical and gas barrier properties. Macromol. Mater. Eng. 298(10), 1065-1073.

Dirrenberger, J., Forest, S. \& Jeulin, D. (2014) Towards gigantic RVE sizes for stochastic fibrous media. Int. J. Solids Struct. 51, 359-376.

Drugan, W. \& Willis, J. (1996) A micromechanics-based nonlocal constitutive equation and estimates of representative volume element size for elastic composites. J. Mech. Phys. Solids 44, 497-524.

Gitman, I., Askes, H. \& Sluys, L. (2007) Representative volume: existence and size determination. Eng. Fract. Mech. 74, 2518-2534.

Hersant, T. \& Jeulin, D. (1976) L'échantillonnage dans les analyses quantitatives d'images. Exemples d'application aux mesures des teneurs de phases dans les agglomérés et des inclusions dans les aciers. Mémoires et Etudes Scientifiques de la Revue de Métallurgie 73, 503.

Hill, R. (1963) Elastic properties of reinforced solids: some theoretical principles. J. Mech. Phys. Solids 11, 357-372.

Huet, C. (1990) Application of variational concepts to size effects in elastic heterogeneous bodies. J. Mech. Phys. Solids 38, 813-841.

Jean, A., Jeulin, D., Forest, S., Cantournet, S. \& N'Guyen, F. (2011). A multiscale microstructure model of carbon black distribution in rubber. J. Microsc. 241(3), 243-260.

Jeulin, D. (2011) Variance Scaling of Boolean Random Varieties. Paris School of Mines, Paris.

Jeulin, D. (2015) Power laws variance scaling of Boolean random varieties. Methodol. Comput. Appl. Probab. in press. 
Kanit, T., Forest, S., Galliet, I., Mounoury, V. \& Jeulin, D. (2003) Determination of the size of the representative volume element for random composites: statistical and numerical approach. Int. J. Solids Struct. 40, 3647-3679.

Kazmierczak, T., Song, H., Hiltner, A. \& Baer, E. (2007) Polymeric onedimensional photonic crystals by continuous coextrusion. Macromol. Rapid Commun. 28(23), 2210-2216.

Kerns, J., Hsieh, A., Hiltner, A. \& Baer, E. (1999) Mechanical behavior of polymer microlayers. Macromol. Symp. 147(1), 15-25.

Lantuejoul, C. (1991) Ergodicity and integral range. J. Microsc. 161(3), 387-403.

Li, X., McKenna, G., Miquelard-Garnier, G., et al. (2014). Forced assembly by multilayer coextrusion to create oriented graphene reinforced polymer nanocomposites. Polymer 55(1), 248-257.

Liu, R., Jin, Y., Hiltner, A. \& Baer, E. (2003) Probing nanoscale polymer interactions by forced-assembly. Macromol. Rapid Commun. 24(16), 943948.

Matheron, G. (1971) The Theory of Regionalized Variables and its Applications. Ecole des Mines de Paris, Paris.

Matheron, G. (1989) Estimating and Choosing. Springer-Verlag, Berlin.

Miquelard-Garnier, G., Guinault, A., Fromonteil, D., Delalande, S. \& Sollogoub, C. (2013) Dispersion of carbon nanotubes in polypropylene via multilayer coextrusion: Influence on the mechanical properties. Polymer 54(16), 4290-4297.

Pelissou, C., Baccou, J., Monerie, Y. \& Perales, F. (2009) Determination of the size of the representative volume element for random quasi-brittle composites. Int. J. Solids Struct. 46, 2842-2855.

Roland, S., Miquelard-Garnier, G., Gervais, M., Guinault, A. \& Sollogoub, C. (2016) Controlling the order of triblock copolymer via confinement induced by forced self-assembly. Mater. Today Commun. 6, 37-43.

Salmi, M., Auslender, F., Bornert, M. \& Fogli, M. (2012) Apparent and effective mechanical properties of linear matrix-inclusion random composites: improved bounds for the effective behavior. Int. J. Solids Struct. 49(10), 1195-1211.

Terada, K., Hori, M., Kyoya, T. \& Kikuchi, N. (2000) Simulation of the multi-scale convergence in computational homogenization approaches. Int. J. Solids Struct. 37, 2285-2311.
Tollar, J. (1966) US Patent No. 3239197.

Wang, H., Keum, J., Hiltner, A. \& Baer, E. (2009) Confined crystallization of PEO in nanolayered films impacting structure and oxygen permeability. Macromolecules 42(18), 7055-7066.

Wang, H., Pietrasanta, A., Jeulin, D., et al. (2015) Modelling mesoporous alumina microstructure with 3D random models of platelets. J. Microsc. 260(3), 287-301.

\section{Appendix A}

Lateral resolution is connected with the vertical resolution limit $\Delta z$. It is the minimum $Z$-coordinate change during scanning, which can be detected at a given noise level. To estimate it, consider $R$ the probing tip curvature radius and $r$ the resolvable surface feature radius (Fig. A.1).

The geometrical analysis allows obtaining the expression for the minimum in-plane detection limit. This is equivalent to determining the chord of an arc with a radius of $R+r$ and its deflection $\Delta z$ :

$$
\Delta x=2 \sqrt{(2(R+r)-\Delta z) \Delta z}
$$

As $\Delta z$ is in the range of the fraction of an angstrom, negligible compared to the radius, the formula can be written as:

$$
\Delta x \approx 2 \sqrt{2(R+r) \Delta z}
$$

Because the best spatial resolution must be the invariant characteristic of the instrument (independent on the studied object), it should be defined from the condition of a flat surface, i.e. $r=0$ such that:

$$
\Delta x=2 \sqrt{2 R \Delta z}
$$

This formula relates in-plane detection limit $\Delta x$, vertical resolution limit $\Delta z$ and tip curvature radius $R$.

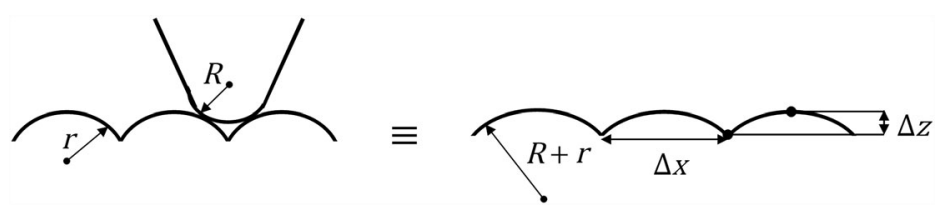

Fig. A.1. Defining $R$ and $r$-curvature radii of tip and resolved objects (left). Expected result of AFM topography study of surface shown in left and defining $\boldsymbol{\Delta} \boldsymbol{z}$ - vertical resolution limit and $\boldsymbol{\Delta} \boldsymbol{x}$ - desired in-plane detection limit (right). 